Open Access

\title{
Quantitative proteomic analysis shows differentially expressed HSPB1 in glioblastoma as a discriminating short from long survival factor and NOVA1 as a differentiation factor between low-grade astrocytoma and oligodendroglioma
}

Marcela Gimenez ${ }^{1}$, Suely Kazue Nagahashi Marie ${ }^{2,4}$, Sueli Oba-Shinjo ${ }^{2}$, Miyuki Uno ${ }^{2}$, Clarice Izumi ${ }^{1}$, João Bosco Oliveira ${ }^{3}$ and Jose Cesar Rosa ${ }^{1 *}$

\begin{abstract}
Background: Gliomas account for more than $60 \%$ of all primary central nervous system neoplasms. Low-grade gliomas display a tendency to progress to more malignant phenotypes and the most frequent and malignant gliomas are glioblastomas (GBM). Another type of glioma, oligodendroglioma originates from oligodendrocytes and glial precursor cells and represents $2-5 \%$ of gliomas. The discrimination between these two types of glioma is actually controversial, thus, a molecular distinction is necessary for better diagnosis.
\end{abstract}

Methods: iTRAQ-based quantitative proteomic analysis was performed on non-neoplastic brain tissue, on astrocytoma grade II, glioblastoma with short and long survival and oligodendrogliomas.

Results: We found that expression of nucleophosmin (NPM1), glucose regulated protein 78 kDa (GRP78), nucleolin (NCL) and heat shock protein $90 \mathrm{kDa}$ (HSP90B1) were increased, Raf kinase inhibitor protein (RKIP/PEBP1) was decreased in glioblastoma and they were associated with a network related to tumor progression. Expression level of heat shock protein 27 (HSPB1/HSP27) discriminated glioblastoma presenting short ( $6 \pm 4$ months, $n=4)$ and long survival ( $43 \pm 15$ months, $n=4)(p=0.00045)$. Expression level of RNA binding protein nova 1 (NOVA1) differentiated low-grade oligodendroglioma and astrocytoma grade II $(p=0.0082)$. Validation were done by Western blot, qRT-PCR and immunohistochemistry in a larger casuistry.

Conclusion: Taken together, our quantitative proteomic analysis detected the molecular triad, NPM1, GRP78 and RKIP participating together with NCL and HSP27/HSPB1 in a network related to tumor progression. Additionally, two new important targets were uncovered: NOVA1 useful for diagnostic refinement differentiating astrocytoma from oligodendroglioma, and HSPB1/HSP27, as a predictive factor of poor prognosis for GBM.

Keywords: Glioma, Network analysis, Isobaric tag, Cancer proteomics, Biomarkers

\footnotetext{
* Correspondence: jcrosa@fmrp.usp.br

'Department Molecular and Cell Biology and Protein Chemistry Center, CTC-Center for Cell Therapy-CEPID-FAPESP-Hemocentro de Ribeirão Preto, Ribeirão Preto Medical School, University of São Paulo, São Paulo, Brazil Full list of author information is available at the end of the article
} 


\section{Background}

Gliomas are the most frequent primary tumors of the central nervous system, accounting for more than $60 \%$ of all brain tumors, and comprise of astrocytomas, oligodendrogliomas, oligoastrocytomas, and ependinomas [1]. Among them, glioblastoma (GBM-grade IV astrocytoma) is the most malignant glioma and despite continuous efforts, the median survival still remains around 15 months after the establishment of diagnosis and the standard care with radiation therapy and chemotherapy with temozolamide [2]. The main study design concerning GBM has aimed to uncover specific drugable targets in signaling pathways with impact in the tumorigenic process and in the extension of overall survival time [3]. In this context, we have recently described two proteins, nucleophosmin (NPM1) and RKIP, involved in RAS/ RAF/MAPK and PI3K/AKT/mTOR pathways [4]. We have also shown that NPM1 knockdown sensitized GBM cell lines to cell death after treatment with temozolamide [5]. Moreover, when NPM1 expression was silenced, expression of GRP78, a member of the heat shock protein 70 involved in protein unfold response, was concomitantly decreased. GRP78 expression was high in GBM, and correlated to cell migration [6]. In the present study we have compared the protein expression profiles of GBM cases presenting short and long survival time, and astrocytoma and oligodendroglioma of different grades of malignancy to further understand the mechanisms of tumor aggressiveness.

Another strategy to understand the rules governing the aggressive behavior of gliomas is to compare astrocytoma to oligodendroglioma, where the latter type of glioma presents a less aggressive clinical evolution. Five and 10 years survival rates for oligodendroglioma are 78 and $51 \%$, respectively, whereas among astrocytoma they are 65 and $31 \%$, respectively [7, 8]. This survival rate difference is due partially to a better response of oligodendroglioma to chemotherapy, including temozolomide or PCV- procarbazin, 1-(2-cloroethyl)-3-cyclohexil-Lnitrosurea and vincristin [9-14] and to radiation therapy $[15,16]$. Therefore, further analysis of differential protein profiles of these glioma types may help to: 1) refine the histopathologic diagnosis, currently based mainly in morphologic characteristics, with large interobserver variability [17, 18], and 2) detect molecular targets that may explain the difference of clinical outcome between low grade astrocytoma and oligodendroglioma.

In this study, we took advantage of isobaric tags for relative and absolute quantification (iTRAQ-8plex) to investigate the proteome related to tumor progression and aggressiveness comparing a set of astrocytoma grade II to oligodendroglioma grade II, and a set of GBM cases presenting short survival $(6 \pm 4$ months, $n=4)$ to GBM cases with long survival $(43 \pm 15$ months, $n=4)$. We have succeeded in uncovering differential protein profiles between these compared sets, highlighting two targets, HSPB1/HSP27 and NOVA1, related to tumor progression and differentiation. Both selected targets were further validated at mRNA expression levels by quantitative PCR, and protein expression and intracellular localization by immunohistochemistry in an independent casuistry of human glioma samples.

\section{Methods}

\section{Tissue processing}

Tissue samples from tumors were collected during surgery and stored at $-80{ }^{\circ} \mathrm{C}$. Tissue samples were microdissected in order to remove areas of necrosis, cellular debris and any non-neoplastic tissue prior to protein, DNA and RNA extraction. The tumor area of interest was concomitantly collected for pathological diagnosis and grade stratification according to the latest WHO classification of CNS tumors by two independent pathologists. The tumors were graded as AST II astrocytoma grade II (AST II), glioblastomas (GBM) and oligodendrogliomas grade II (OLI II) and oligodendrogliomas grade III (OLI III). GBMs were divided in two subgroups based on patients' overall survival time after diagnosis as GBM of short survival (GBM-SS, $6 \pm 4$ months, $n=4$ ) and long survival (GBMLS, $43 \pm 15$ months, $n=4)$. Non-neoplastic brain tissues $(\mathrm{NN}$, mean age at surgery, $29 \pm 7$ years, $n=4)$ were obtained from individuals submitted to temporal lobe resection for epilepsy surgery and examined by a pathologist who confirmed the abundance of astrocytic cells in the resected tissue. Four samples for each group were pooled and analysed by the proteomic approach (ASTII mean age at diagnosis, $33 \pm 7$ years; GBM-SS $48 \pm 23$ years; GBM-LS $48 \pm 18$ years; OLI II $42 \pm 16$ years and OLI III $48 \pm 15$ years). An independent casuistry comprised of 22 (NN), 23 (AST I), 26 (AST II), 18 (AST III), 83 (AST IV or GBM), 25 (OLI II), and 26 (OLI III) was analyzed at the validation step by qRT-PCR for the selected targets. All samples were collected during surgical procedures by the Neurosurgery Group of the Department of Neurology at the Hospital das Clinicas of School of Medicine of São Paulo, University of Sao Paulo, Brazil from 2000 to 2008 and the follow-up of cases are being carried out to date. This study was approved by the Brazilian National Bioethics Commission (CONEP), and by the Ethics Committee of the Medical School of Ribeirao Preto and School of Medicine of São Paulo of the University of Sao Paulo. Written consent was obtained from each patient authorizing the use of their tissues in the present investigation.

\section{Tumor protein extraction}

Tissue samples were mechanically homogenized in lysis buffer containing $30 \mathrm{mM}$ Tris- $\mathrm{HCl} \mathrm{pH} \mathrm{7.5,} 150 \mathrm{mM}$ $\mathrm{NaCl}, 1$ \% Triton $\mathrm{X}-100,10 \%$ glycerol and a protease 
inhibitor cocktail. The cell lysates were centrifuged at 20,000 g for $30 \mathrm{~min}$, the supernatants were precipitated with $20 \%$ trichloroacetic acid and washed three times with cold acetone. Electrophoresis buffer $(200 \mu \mathrm{L})$ containing $10 \mathrm{mM}$ Tris base, $\mathrm{pH}$ 9.0, $7 \mathrm{M}$ urea, $2 \mathrm{M}$ thiourea, $65 \mathrm{mM}$ DTT and $4 \%$ CHAPS was added to each pellet. Proteins pellets were then submitted to three cycles of 5 min each in an ultrasound bath (UltraSonic Clear 750, UNIQUE) centrifuged and supernatant were kept for protein concentration determination.

\section{Sample preparation and ITRAQ labeling}

Each protein extract of tumor and non-neoplastic tissue were quantified by the method of Bradford [19]. Twenty five $\mu \mathrm{g}$ of each patient sample was pooled to normalize $100 \mu \mathrm{g}$ total protein for each category. Additional file 1: Figure S1 describes a schematic experimental approach. Pooled samples were mixed with $6 \times$ volume of cold acetone $\left(-20{ }^{\circ} \mathrm{C}\right)$ and incubated for $60 \mathrm{~min}$ at $-20{ }^{\circ} \mathrm{C}$. The proteins pellets were reconstituted according to manufacturer's protocol (Applied Biosystems, Framingham, MA, USA). Briefly, proteins pellets were resuspended into $20 \mu \mathrm{L}$ of dissolution buffer $(0.5 \mathrm{M}$ triethylammonium bicarbonate), $1 \mu \mathrm{L}$ denaturant (2\% SDS), and $2 \mu \mathrm{L}$ reducing reagent (50 mM tris-(2-carboxyethyl) phosphine). Free cysteine was blocked by adding $1 \mu \mathrm{L}$ of $200 \mathrm{mM}$ methyl methanethiosulfonate in isopropropanol. Sequencing grade modified trypsin was from Promega (Madison, WI) and was reconstituted with deionized water at $1 \mu \mathrm{g} / \mu \mathrm{L}$ concentration. In each vial $10 \mu \mathrm{L}$ of trypsin solution was added and incubated overnight $(18 \mathrm{~h})$ at $37{ }^{\circ} \mathrm{C}$. Reagents of 8 plex iTRAQ were allowed to reach room temperature and then reconstituted with $50 \mu \mathrm{L}$ of isopropanol. Each label reagent was mixed with the corresponding protein digest and incubated at room temperature for $2 \mathrm{~h}$. Samples were pooled into a new vial and dried in SpeedVac (Savant Inc, New York, NY). After reconstituted with $0.1 \%$ formic acid (FA), the digest was desalted on a Waters Oasis HLB column and eluted with $60 \%$ acetonitrile $(\mathrm{ACN}) / 0.1 \%$ FA. Eluted peptide mixture was dried.

\section{Strong cation exchange fractionation (SCX)}

The sample was reconstituted with $100 \mu \mathrm{L}$ SCX buffer A $\left(10 \mathrm{mM} \mathrm{KH}_{2} \mathrm{PO}_{4}, 20 \% \mathrm{ACN}, \mathrm{pH} 2.7\right)$ and separated on a PolyLC Poly-sulfoethyl-A column (200x2.1 mm, $5 \mu \mathrm{m}$, $200 \AA$ ) with a linear $200 \mu \mathrm{L} / \mathrm{min}$ gradient of $0-70 \%$ buffer B $\left(10 \mathrm{mM} \mathrm{KH_{2 }} \mathrm{PO}_{4}, 20 \% \mathrm{ACN}, 500 \mathrm{mM} \mathrm{KCl}\right.$, $\mathrm{pH} 2.7$ ) in $45 \mathrm{~min}$ on an Agilent 1200 LC device with Chemstation B.02.01 control software. Fractions were collected each minute and eventually pooled into 20 fractions. The fractions were desalted, eluted, and dried as described above using Waters Oasis HLB column.

\section{Mass spectrometry}

The samples were reconstituted with $0.1 \%$ formic acid. Liquid chromatography was performed on an Eksigent nanoLC-Ultra 1D plus system (Dublin, CA). Peptide digest was first loaded on a Zorbax 300SB-C18 trap (Agilent, Palo Alto, CA) at $6 \mu \mathrm{L} / \mathrm{min}$ for $5 \mathrm{~min}$, then separated on a PicoFrit analytical column (100 mm long, ID $75 \mu \mathrm{m}$, tip ID $10 \mu \mathrm{m}$, packed with BetaBasic $5 \mu \mathrm{m}$ $300 \AA$ particles, New Objective, Woburn, MA) using a 40-min linear gradient of $5-35 \% \mathrm{ACN}$ in $0.1 \% \mathrm{FA}$ at a flow rate of $250 \mathrm{~nL} / \mathrm{min}$. Mass analysis was carried out on an LTQ Orbitrap Velos (Thermo Fisher Scientific, San Jose, CA) with data-dependent analysis mode, where MS1 scanned full MS mass range from m/z 300 to 2000 at 30,000 mass resolution and six HCD MS2 scans were sequentially carried out at resolution of 7500 with $45 \%$ collision energy, both in the Orbitrap.

\section{Database search and quantitative data analysis}

MS/MS spectra from 20 fractions were searched against the Swiss Prot (Swiss Institute of Bioinformatics) database, taxonomy Homo sapiens (human) using Mascot software (Matrix Science, London, UK; version 2.3), with precursor mass tolerance at $20 \mathrm{ppm}$, fragment ion mass tolerance at $0.05 \mathrm{Da}$, trypsin enzyme with 2 miscleavages, methyl methanethiosulfonate of cysteine and iTRAQ 8plex of lysine and the $\mathrm{n}$-terminus as fixed modifications, and deamidation of asparagine and glutamine, oxidation of methionine and iTRAQ 8plex of tyrosine as variable modifications. The resulting data file was loaded into Scaffold Q+ (version Scaffold 4.3.0, Proteome Software Inc., Portland, OR) to filter and quantitate peptides and proteins. Peptide identifications were accepted at $80.0 \%$ or higher probability as specified by the Peptide Prophet algorithm [20] and a false discovery rate (FDR) of less than $1 \%$. Protein identifications were accepted at $95.0 \%$ or higher probability and contained at least 2 identified peptides with FDR less than $1 \%$. Protein probabilities were assigned by the Protein Prophet algorithm [21]. Proteins that contained similar peptides and could not be differentiated based on MS/MS analysis alone were grouped to satisfy the principles of parsimony. Peptides were quantified as the centroid reporter ion peak intensity, with minimum of $5 \%$ of the highest peak in the spectrum. Intra-sample channels were normalized based on the median ratio for each channel across all proteins. Isobaric tag sample was normalized by comparing the median protein ratios for the reference channel. Quantitative protein values were derived from only uniquely assigned peptides. Protein quantitative ratios were calculated as the median of all peptide ratios. Standard deviations were calculated as the interquartile range around the median. Quantitative ratios were $\log _{2}$ normalized for final quantitative testing. 


\section{Western blot}

The samples were diluted in NuPAGE SDS Sample buffer (Invitrogen NP0007) and the SDS-PAGE was performed using NuPAGE Novex Bis-Tris Mini Gels 4-12\%. SDS-PAGE gels were electrobloted in iBlot Device and the membranes were incubated with primary antibodies HSPB1/HSP27 and HSP90B1(GRP94) from Cell Signaling Technology; NPM and RKIP from Zymed-Invitrogen; NCL and $\beta$-actin from Santa Cruz Biotechnology; NOVA-1 from Sigma-Aldrich. The same source of antibodies HSPB1 and NOVA1 were used for immunohistochemistry.

\section{RNA extraction and CDNA synthesis}

Total RNA was extracted from each tissue using the RNeasy Mini Kit (Qiagen, Hilden, Germany). RNA quantification and purification was determined by measuring absorbance at 260 and $280 \mathrm{~nm}$. A260/A280 ratios in the 1.8-2.0 range were considered to indicate a satisfactory level of purity. Denaturing agarose gel electrophoresis was used to assess the quality of the samples. cDNA synthesis was performed by reverse transcription of $1 \mu \mathrm{g}$ total RNA previously treated with one unit of DNase I (FPLC-pure, GE Healthcare, Piscataway, NJ,) using random and oligo(dT) primers, RNase inhibitor, and SuperScript III (Life Technologies) according to the manufacturer's recommendations.

\section{Quantitative real-time PCR (qRT-PCR)}

For qRT-PCR, quantitative data were normalized relative to the internal housekeeping control genes hypoxanthine phosphoribosyltransferase 1 (HPRT), beta-glucuronidase (GUSB), and TATA-box binding protein (TBP) [22]. The geometric mean of the housekeeping genes was used for the analysis of relative expression of tissue samples. Primer sequences were as follows $\left(5^{\prime}-3^{\prime}\right)$ : HSPB1 F: GGACGAGCTGACGGTCAAGA, HSPB1 R: CGGGA GATGTAGCCATGCT, NOVA1 F: GGAGCCACCATC AAGCTGTCTA, NOVA1 R: TCAGTGCTTCAACCGT TCCCT, HPRT F: TGAGGATTTGGAAAGGGTGT, HPRT R: GAGCACACAGAGGGCTACAA, GUSB F: A AAATACGTGGTTGGAGAGCTCATT, GUSB R: CCG AGTGAAGATCCCCTTTTTA, TBP F: AGGATAAGA GAGCCACGAACCA, and TBP R: CTTGCTGCCAGT CTGGACTGT synthesized by IDT. Sybr Green I amplification mixtures $(12 \mu \mathrm{L})$ contained $3 \mu \mathrm{L}$ cDNA, $6 \mu \mathrm{L}$ $2 \times$ Power Sybr Green I Master Mix (Applied Biosystems, Foster City, CA), and forward and reverse primers at final concentrations of 200-400 nM. Reactions were run on an ABI 7500 Real-Time PCR System (Applied Biosystems). The cycling conditions were: incubation at $50{ }^{\circ} \mathrm{C}$ for $2 \mathrm{~min}$ to activate UNG, initial denaturation at $95{ }^{\circ} \mathrm{C}$ for $10 \mathrm{~min}$, and 40 cycles of $15 \mathrm{~s}$ each at $95{ }^{\circ} \mathrm{C}$ and at $60{ }^{\circ} \mathrm{C}$ for $1 \mathrm{~min}$. DNA melting curve analysis showed a single peak for all genes. The $2^{-\Delta \Delta C T}$ equation was applied to calculate the relative expression [23]. For the relative expression analysis of GBM cases, the mean of control nonneoplastic brain samples was used as calibrator.

\section{Immunohistochemistry}

For immunohistochemical detection of HSPB1 and NOVA1, tissue sections were routinely processed and subjected to antigen retrieval. Briefly, slides were immersed in $10 \mathrm{mM}$ citrate buffer, $\mathrm{pH} 6.0$ and incubated at $122{ }^{\circ} \mathrm{C}$ for $3 \mathrm{~min}$ using an electric pressure cooker (BioCare Medical Walnut Creek, CA). Specimens were then blocked and further incubated with a mouse monoclonal antibody raised against human HSPB1 and NOVA1 at a final dilution of $1: 100$ at $16-20{ }^{\circ} \mathrm{C}$ for $16 \mathrm{~h}$. The reaction was developed using a Novolink commercial kit (Novocastra, New Castle, UK) at room temperature using diaminobenzidine, and Harris hematoxylin for nuclear staining. All prepared slides were independently analyzed by two observers, and the positive reaction was quantitated for HSPB1 and NOVA1 as the percentage of positive cytoplasm/nuclei cells: zero (0), when no positivity was detected; 1 , when up to $25 \%$ of positive cells were present; 2 , for $26-50 \%$ of positive cells; 3 , for $51-75 \%$ of positive cells, and 4 , for over $76 \%$ of positive cells.

\section{Statistical analysis}

The statistical analysis of HSBP1 and NOVA1 expression by qRT-PCR in astrocytomas, oligodendrogliomas and non-neoplastic tissues was performed by Kruskal-Wallis and Mann-Whitney tests as well as the proteomic profiling through statistical package included in Scaffold v.4.3.0 software, blocked $t$-test and ANOVA for categories (p-value, ASTRO, OLI or ASTRO/OLI) (Proteome Software, Inc, Portland, Oregon). Discrimination of variables was calculated by the receiver operator characteristic (ROC) curve utilizing area under curve and asymptotic significance. The continuous variables were categorized through a curve using ROC the value with the best sensitivity and specificity. Differences in gene and protein expressions were considered to be statistically significant at $p<0.05$.

\section{Results}

Identification of proteins differentially expressed in gliomas using isobaric tags for relative and absolute quantification (iTRAQ)

Proteomic analysis using iTRAQ isobaric tags was performed using pool of samples from astrocytoma grade II (AST II), glioblastoma (GBM) sub-grouped into cases presenting short and long survival after diagnosis (GBM-SS, $6 \pm 4$ months, $n=4$ and GBM-LS43 \pm 15 months, $n=4$, respectively), oligodendroglioma grade II (OLI II) and 
oligodendroglioma grade III (OLI III). The proteins were selected and quantified in Scaffold software v.4.3.0 (Fig. 1 and Table 1). Proteins were differentially expressed when compared to non-neoplastic tissue $(\mathrm{NN})$ as the ratio was above or below Log2 Fold Change $(0.6=1.5$-fold $)$ and statistically significant between categories. The results of the following sets were compared: 1) AST II vs. GBMs, and OLI II vs. OLI III to address protein involved in tumor malignant progression; 2) GBM-SS vs. GBM-LS to address proteins involved in prognosis; 3) AST II vs. OLI II to address proteins involved in the differentiation between these two low grade gliomas with impact in tumor aggressiveness. We were able to identify 1095 proteins labeled with iTRAQ and using minimum of 2 peptides per protein (Additional file 3: Table S1 - Protein report and Additional file 4: Table S2 Peptide report), which 268 presented difference of expression in at least one group (Additional file 5: Table S3 Protein ratio). The

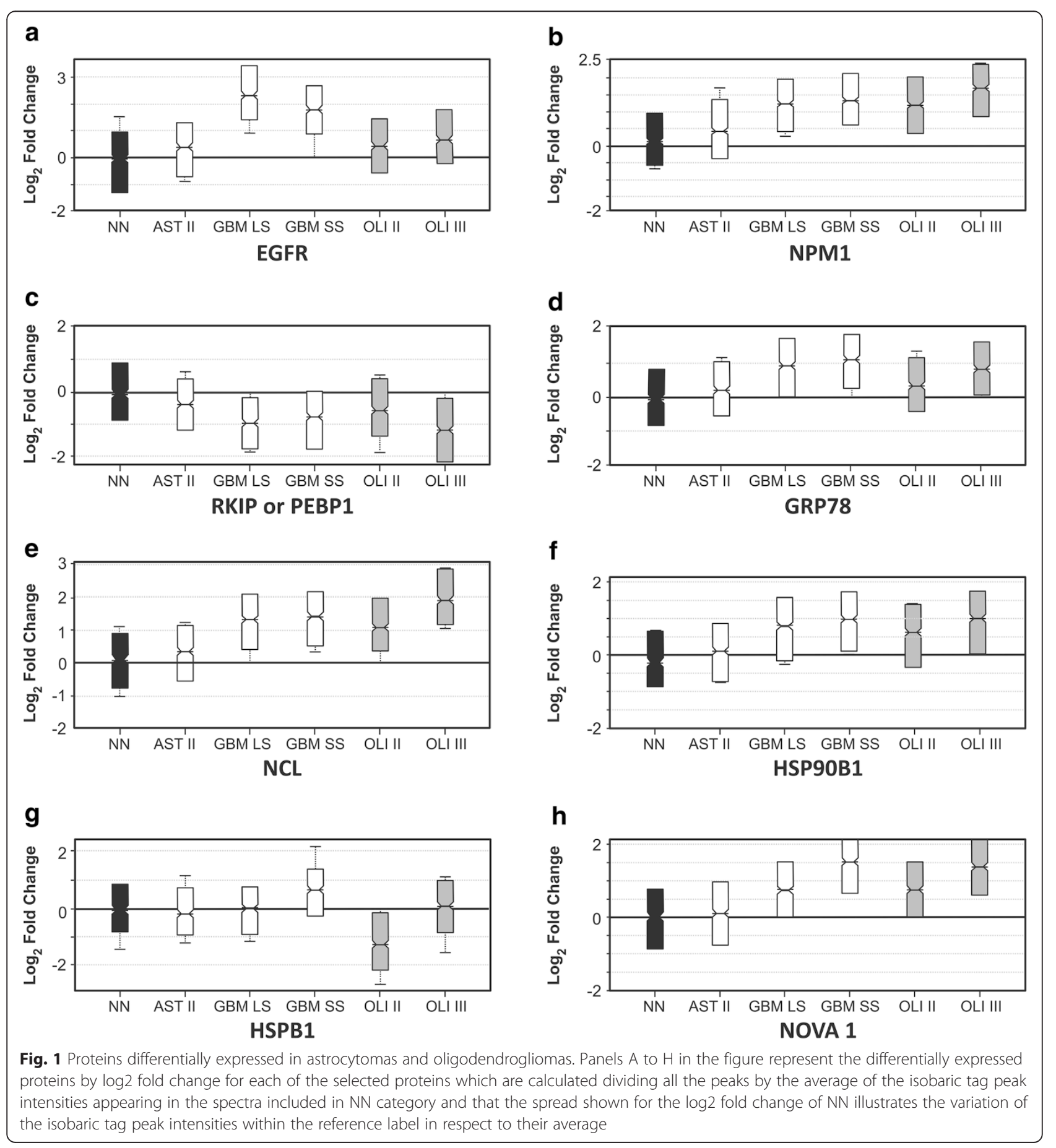


Table 1 Selected proteins from quantitative proteomic analysis of astrocytomas and oligodendriomas tumor samples $(n=4)$. Proteins are expressed as log2 fold change in relation to non-neoplastic brain tissue (NN)

\begin{tabular}{|c|c|c|c|c|c|c|c|c|c|c|c|c|}
\hline Id Protein & Acc & NN & AST II & GBM SS & GBM LS & OLI ॥ & OLI III & $\begin{array}{l}\text { Spectra } \\
\text { Count }\end{array}$ & $\begin{array}{l}\% \text { Seq } \\
\text { Cov }\end{array}$ & $\begin{array}{l}\text { blocked } \\
\text { t-test } \\
\text { (p-value } \\
\text { ASTRO) }^{a}\end{array}$ & $\begin{array}{l}\text { blocked } \\
\text { t-test } \\
\text { (p-value } \text { OLI) } \\
\text { b }\end{array}$ & $\begin{array}{l}\text { blocked } \\
\text { ANOVA } \\
\text { test (p-value } \\
\text { ASTRO/OLI) } \\
\end{array}$ \\
\hline $\begin{array}{l}\text { Epidermal growth factor } \\
\text { receptor }\end{array}$ & EGFR & Ref & 0.2 & 1.5 & 2.2 & 0.3 & 0.6 & 20 & 13.2 & 0.0023 & 0.93 & $<0.0001$ \\
\hline Nucleophosmin & NPM1 & Ref & 0.2 & 1.1 & 1.1 & 0.8 & 1.3 & 14 & 32.3 & 0.0049 & $<0.0001$ & $<0.0001$ \\
\hline $\begin{array}{l}78 \mathrm{kDa} \text { glucose-regulated } \\
\text { protein }\end{array}$ & GRP78 (HSPA5) & Ref & 0.3 & 1.1 & 0.9 & 0.2 & 0.6 & 44 & 43.0 & $<0.0001$ & 0.0008 & 0.037 \\
\hline $\begin{array}{l}\text { Phosphatidylethanolamine- } \\
\text { binding protein } 1\end{array}$ & PEBP1 (RKIP) & Ref & -0.4 & -1.0 & -1.1 & -0.6 & -1.4 & 36 & 79.1 & $<0.0001$ & $<0.0001$ & $<0.0001$ \\
\hline Heat shock protein beta-1 & HSPB1 & Ref & 0.1 & 1.6 & 0.8 & 0.3 & 0.5 & 9 & 49.8 & 0.00045 & 0.89 & 0.28 \\
\hline RNA-binding protein Nova-1 & NOVA1 & Ref & 0.1 & 0.9 & 2.2 & 0.8 & 1.3 & 3 & 7.8 & 0.120 & 0.011 & 0.0082 \\
\hline Endoplasmin & (ENPL) HSP90B1 & Ref & 0.2 & 1.0 & 0.9 & 0.6 & 1.0 & 44 & 25.8 & $<0.0001$ & $<0.0001$ & $<0.0001$ \\
\hline Nucleolin & $\mathrm{NCL}$ & Ref & 0.2 & 1.3 & 1.1 & 1.0 & 1.9 & 27 & 25.1 & $<0.0001$ & $<0.0001$ & $<0.0001$ \\
\hline
\end{tabular}

Statistical test for ratio-based normalization of isobaric tags (Scaffold v.4.3.0) - The samples were grouped as follows:

a astrocytomas = NN, AST II, GBM-SS and GBM-LS

boligodendroglioma $=\mathrm{NN}, \mathrm{OLI}$ II and OLI III

cstrocytoma and oligodendroglioma $=$ NN, AST II and OLI II

gene ontology analysis revealed that proteins differentially expressed were mainly involved with metabolic processes, biological processes regulation and binding to proteins, RNA and nucleotides.

\section{Selection and validation of proteins involved in tumor malignant progression}

Proteins selected as having alteration of expression and known to participate in the process of tumor progression are shown in Table 1 and Fig. 1. NPM1, RKIP/ PEBP1 and GRP78 expressions were significantly distinct in GBMs and oligodendrogliomas compared to AST II and $\mathrm{NN}$ ( $p<0.0001$, blocked $t$ test), corroborating previous data of our group [4-6]. Particularly, NPM1 expression presented correlation with tumor malignant progression as lower expressions were observed in non-neoplastic tissue, AST II and OLI II compared to the expressions of GBM and OLI III. Interestingly, phosphatidylethanolaminebinding protein 1 (PEBP1), also known as raf kinase protein inhibitor (RKIP), was decreased in high grade gliomas in relation to the non-neoplastic tissue and lower grade gliomas, as previously demonstrated by our group [4]. These reproduced data related to NMP1, GRP78 and RKIP demonstrated the consistency of the proteomics results herein presented by iTRAQ methodology. Also, EGFR was highly expressed in GBM compared to AST II, as expected, however, similar results were not observed for OLI II and III $(p=0.930)$ (Fig. 1a). Selected proteins as NPM1, RKIP, HSP90B1 and NCL were also differentially expressed among the analyzed subgroups and these levels of expression were validated by western blotting of pooled samples (Fig. 2). GRP78 was previously validated elsewhere $[5,6]$.

HSPB1 (HSP27) as a predictive factor between GBM cases with short and long overall survival time

The most interesting differentially expressed protein was heat shock protein beta-1 (HSPB1) (Fig. 1g, Table 1) that

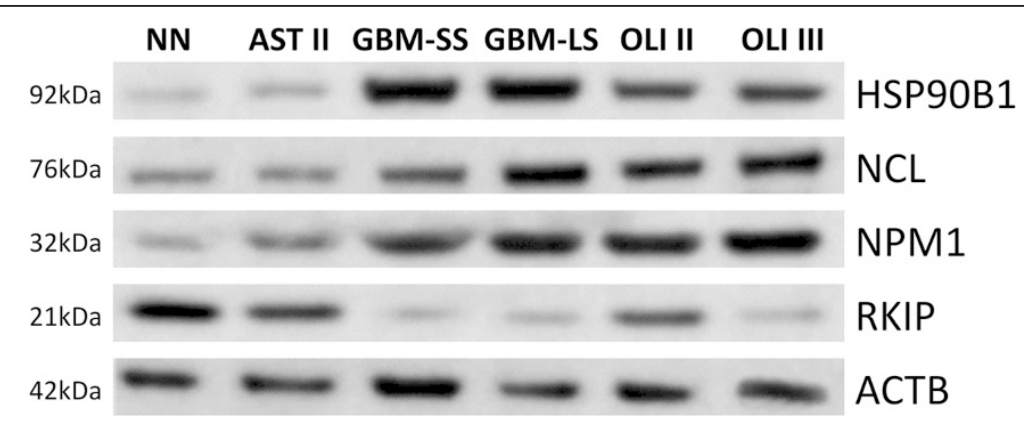

Fig. 2 Western blot validation of differentially expressed proteins in patient pools of astrocytomas and oligodendrogliomas. The same pool of patient sample used for quantitative proteomics was used for validation by immunodetection. The assay was normalized to actin 
was highly expressed in GBM-short survival (GBM-SS), especially when compared to GBM-long survival (GBMLS) $(p=0.00045)$. This finding was also observed at the HSPB1 mRNA expression level, where its expression was significantly different among GBM patients who presented less than 12 months of survival time compared to those presenting more than 16 months survival ( $p=0.0287$, Mann Whitney test). HSPB1 expressions were still distinct when GBMs cases with less than 12 months and more than 24 months survivals were compared ( $p=0.0816$, Mann Whitney test) (Fig. 3a and b). Statistical significance would be reached increasing the number of observations of GBM cases presenting overall survival time longer than 24 months, a very rare condition. Additionally, a stepwise increase of HSPB1 mRNA expression was observed in parallel to the increase in malignancy mainly in diffusely infiltrative astrocytomas from grade II to IV ( $p<0.05$ to $p<0.0001$, Kruskal Wallis and Dunn's tests), and when these results were plotted on ROC curves, an increasing value of the area in parallel to the increment of the malignancy was observed (Fig. 4a), strongly suggesting that HSPB1

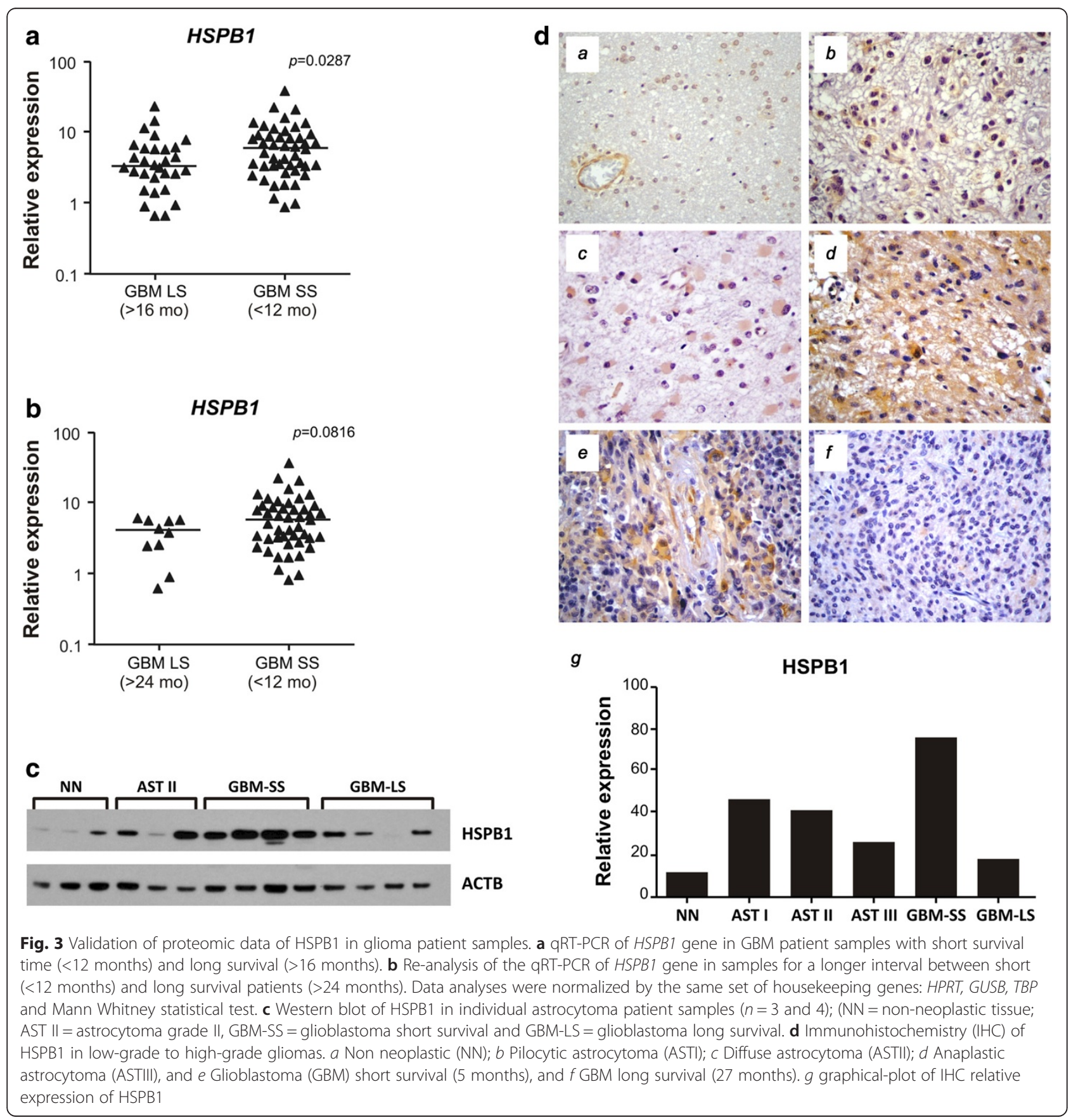




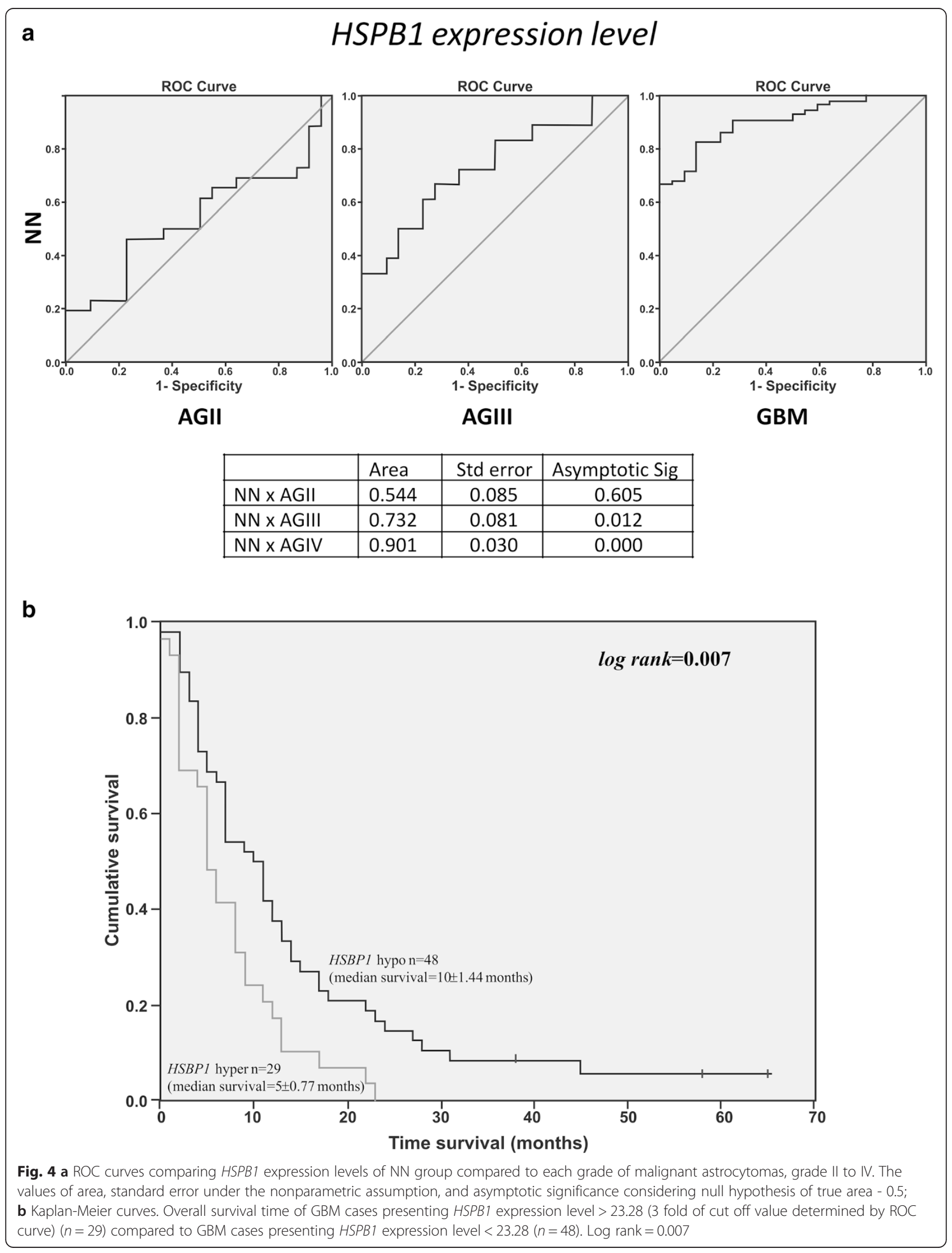


expression level as an indicator of tumor progression. Moreover, when the overall survival times of GBM cases presenting \pm 3 fold cut off value of HSPB1 expression level calculated based on the ROC curve $(3 \times 7.76=23.28)$ were compared to those presenting HSPB1 expression level $<23.28$, it resulted in a Kaplan-Meier curve with log rank of 0.007 (Fig. 4b). This finding was independent to the IDH1 mutation status [24], according to multivariate proportional hazards analysis (Cox model), where HSPB1 expression status (hyper and hypo expression) presented hazard ratio (HR) of 1.86 with $95 \%$ confidence interval $(\mathrm{CI})$ ranging from $1.14 \pm 3.03$, and $p$ value of 0.012 . On the other hand, IDH1 mutation status (mutated IDH1 compared to wild type) presented $\mathrm{HR}=1.35,95 \% \mathrm{CI}=0.64 \pm 2.84, p=0.43$. Similar analysis was not feasible to MGMT methylation status, as such results were available for only 51 out of 83 GBM cases due to limitation of biological sample. Nevertheless, we have previously reported no impact of MGMT methylation status on the overall survival time among GBM cases of this series (log rank, Mantel-Cox $=0.204$ ) [25]. HSPB1 protein expression levels of GBM cases with short and long survival were validated individually by western blot analysis, which showed more intense immunostaining of protein in GBM cases with short survival, confirming the possible usefulness of HSPB1 as predictive factor of worse prognosis (Fig. 3c). HSPB1 protein expression was further confirmed by immunohistochemistry in astrocytoma samples, comprising pilocytic astrocytoma (grade I), low grade astrocytoma (grade II), anaplastic astrocytoma (grade III) and GBM (grade IV) with short (5 months) and long survival (27 months), and in non-neoplastic brain tissue (Fig. 3d). High abundance of HSPB1 was detected in grade IV astrocytoma, particularly in GBM-SS (5 months, Fig. $3 \mathrm{~d}(e)$ ) with unequivocal contrast to the weak labeling of a GBM-LS case (27 months, Fig. $3 \mathrm{~d}(f))$. Graph of immunochemistry of HSPB1 was demonstrated in Fig. $3 \mathrm{~d}(g)$, showing that GBM-SS is highly positive in contrast to GBM-LS. These results of proteomics, gene and protein expressions allow to elect HSPB1 as a predictive factor of tumor aggressiveness in a restricted set of GBM cases, and it may worth further exploration as a potential therapeutic target for these specific cases.

\section{NOVA1 as a differentiation factor between Low grade astrocytoma and oligodendroglioma}

RNA binding protein nova 1 (NOVA1) presented an interesting expression profile when low grade astrocytomas and oligodendrogliomas grade II were compared (Table $1, p=0.0082$ ). qRT-PCR for NOVA1 showed a significant difference between OLI II and AST II $(\mathrm{p}<0.0005$,
Mann Whitney test) (Fig. 5a). NOVA1 was validated by western blot through the analysis of patients of grade II astrocytoma and oligodendroglioma II and III individually. The results showed heterogeneity in the immunodetection of NOVA1 and at least one case, the protein was not detected, from a total of 4 samples in OLI II (Fig. 5b). However, NOVA1 immunohistochemistry was highly concordant with NOVA1 at mRNA level and proteomic profiling, showing a major concentration of this protein in nuclei compartment (Fig. 5c). NOVA1 can be used as a molecular marker to differentiate low-grade astrocytoma from low-grade oligodendroglioma, and therefore, may be helpful for the refinement of the diagnosis currently based mainly on histopathological characteristics.

\section{Network analysis of molecular triad NPM1, RKIP and GRP78 using metacore}

The network analysis of three molecules NPM1, RKIP and GRP78 by MetaCore program allowed the addition of two proteins in the context of systems biology, HSPB1 (HSP27) and nucleolin (NCL), interconnected to at least three transcription factors, ESR1, STAT3 and SP1, and downstream of EGFR receptor pathway, classically known as modified in GBM (Fig. 6). The results of this network analysis highlight the importance of several proteins found in this work to be altered in the tumor samples and will be discussed later in the next section.

\section{Discussion}

One of the most productive and direct way of obtaining information about the development of diseases such as cancer, especially brain cancer, is the combination of genomic and proteomic strategies of tumor specimens taken from patients. Each tumor sample provides new markers of the disease and improves the knowledge about the biology of tumors. One of most important findings of our report is the detection of NOVA1 as differentiation factor between low-grade astrocytoma and oligodendroglioma. The clinical impact of such a diagnostic refinement based on molecular marker is relevant as astrocytoma presents more aggressive progression than oligodendroglioma, and accordingly a diagnosis of an astrocytic tumor requires more aggressive therapeutic strategies. NOVA1 is an alternative splicing factor involved in the main mechanism of increasing proteome diversity coded by a limited number of genes. Together with other splicing factors, including ESRP1 and 2, MBNL1, PTBP1, and RBFOX2, NOVA1 contributes to establishing a cell type-specific splicing programs [26]. The role of altered expression of NOVA1 in glioma is still unknown. In oligodendrogliomas, $\mathrm{Xu}$ et al. [27] have reported the expression of multiple larger-sized transcripts for several genes attributed to hnRNP A1, a component of the 

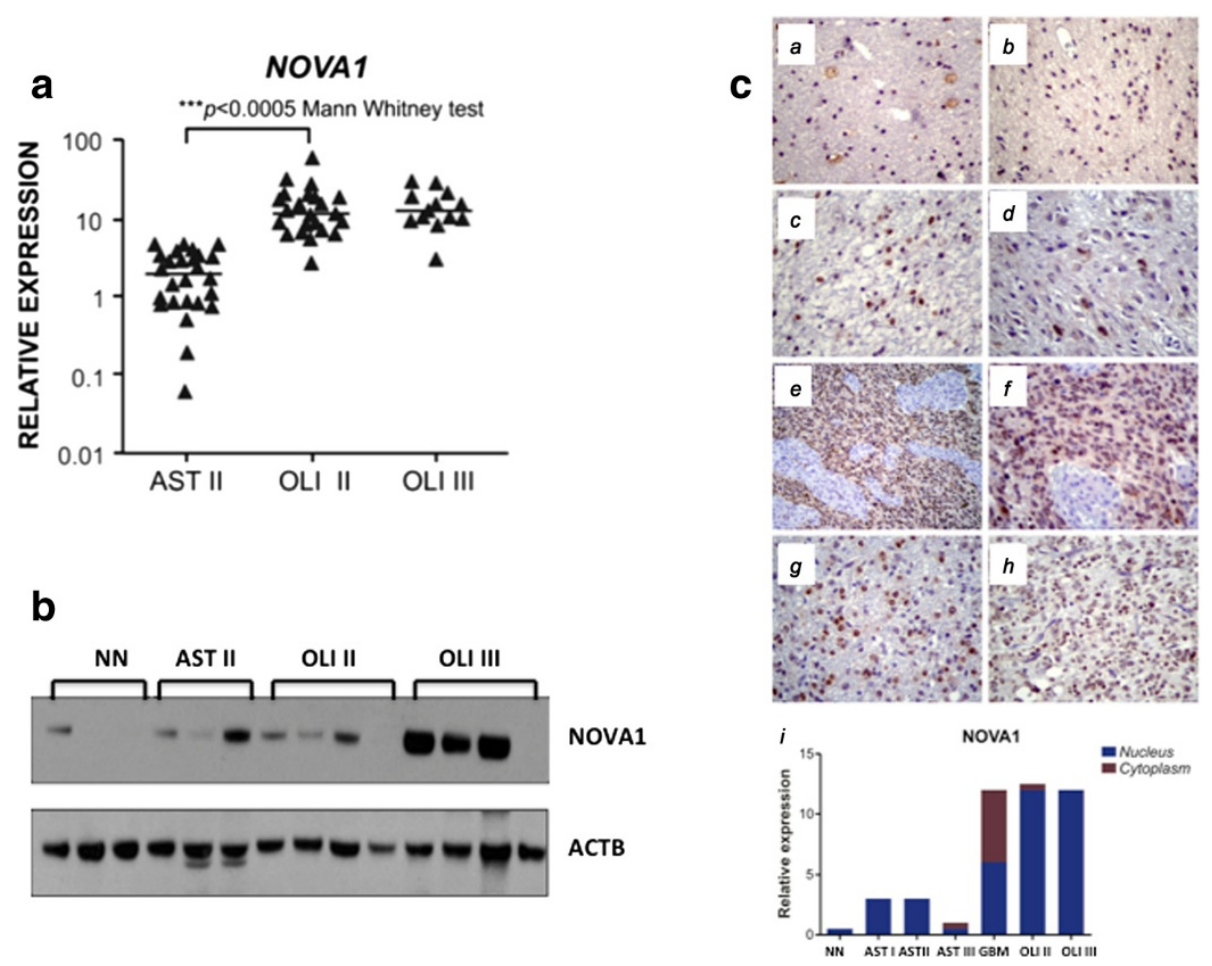

Fig. 5 Validation of proteomic data of NOVA1 in low-grade astrocytoma and oligodendroglioma patient samples. a qRT-PCR of NOVA1 gene in astrocytoma grade II (AST II) and oligodendroglioma (OLI II and OLI III). Data analyses were normalized by same set of housekeeping genes: HPRT, GUSB, TBP and Mann Whitney statistical test. $\mathbf{b}$ Western blot of NOVA1 in individual astrocytoma and oligodendroglioma patient samples $(n=3$ and 4); c Immunohistochemistry (IHC) of NOVA1 in astrocytomas and oligodendrogliomas. a Non neoplastic (NN); $b$ Pilocytic astrocytomas (AST I); c Diffuse astrocytoma (AST II); $d$ Anaplastic astrocytomas (AST III), and e Glioblastoma (GBM, 200x) f Glioblastoma (GBM, 400x); $g$ Oligodendroglioma II and h Oligodendroglioma III. i Graphical plot of IHC relative expression of NOVA1 distribution between nucleus and cytoplasm

spliceosome, which rules directly the selection of splice site leading to a preferential expression of larger-sized transcripts. These authors have suggested that the expression of large transcript could be useful for distinguishing oligodendroglial from astroglial gliomas [27]. In the present study, NOVA1 expression profile has proved to also differentiate oligodendroglioma from astrocytoma, and NOVA1 higher expression in oligodendroglioma may contribute to the preferential splicing program in this type of glioma.

In this work, we also detected and validated HSPB1 (HSP27) as a predictive factor for poor prognosis in GBM. High expression of HSPB1 was demonstrated in GBM cases with survival time shorter than 12 months. HSPB1 is a multifunctional protein that is dependent of oligomerization and phosphorylation status [28]. HSPB1/ HSP27 is a human small heat shock protein, a chaperone that regulates fundamental cellular processes in normal unstressed cells as well as in many cancer cells, including breast, ovarian, endometrial cancers, osteosarcoma, and leukemia [29]. HSPB1/HSP27 is constitutively expressed at low levels in many cells and tissues, and its increased expression level has been correlated to the enhancement of cellular resistance, even in the presence of DNA damage due to UV radiation. Recently, the switch between apoptosis and survival, modulated by Akt stability, has been attributed to HSPB1/HSP27 in adenocarcinoma cells [30]. Interestingly, a network analysis by MetaCore ${ }^{\text {Tx }}$ has demonstrated that three targets, NPM1, GRP78 and RKIP, previously published by our group [4-6], are associated with the other two targets unveiled in the present combined analysis of proteomics and oligonucleotide expression arrays: HSPB1/HSP27 and nucleolin (NCL) demonstrated in Fig. 6. HSPB1/HSP27 is downstream of the canonical activation of EGFR through the transcription factor, nuclear estrogen receptor 1 (ESR1) [31]. Nucleolin (NCL) is a nucleolar phosphoprotein involved in the synthesis and maturation of ribosomes, found associated with intranuclear chromatin and pre-ribosomal particles, which induces chromatin decondensation by binding to histone H1. NCL plays a role in pre-rRNA transcription and ribosome assembly in the process of transcriptional elongation. NCL is downstream to RKIP in a canonical pathway, where RKIP down-regulates BRAF as demonstrated in melanoma cancer cells [32]. BRAF negatively regulates AKT [33] through directly binding to Rictor (mTORC2) [34]. AKT phosphorylates MYT1 kinase and decreases its activity [35], and the latter 


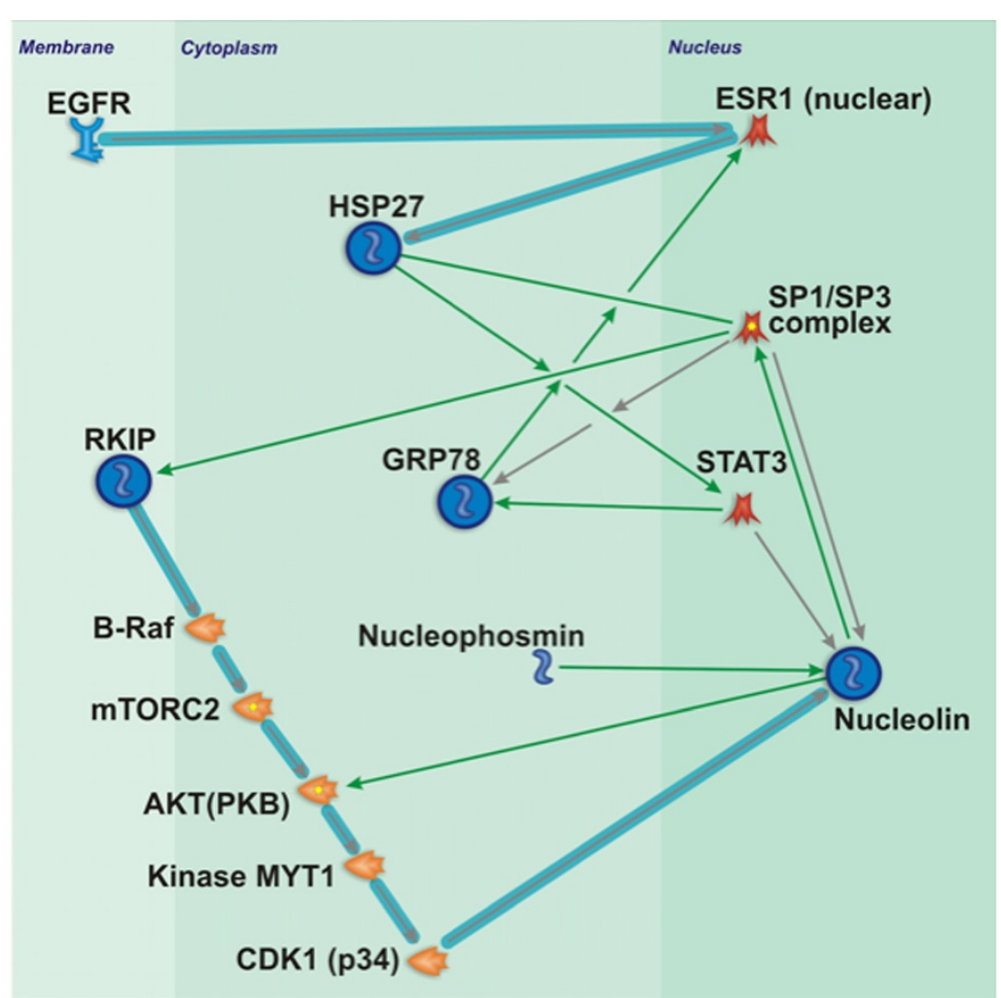

Fig. 6 MetaCore analysis of differential modulated proteins in glioma tumors. The NPM1, RKIP, GRP78, HSP27 (HSPB1) and NCL proteins were subjected to network interactions using the MetaCore software and placed in a context of systems biology implying important findings to contribute to the progress of studies in glioma tumorigenesis and their participation in canonical pathways. See MetaCore symbol legend in Additional file 2: Figure S2

phosphorylates CDK1 and also decreases its activity [36]. CDK1 maintains NCL stability via phosphorylation during mitosis [37]. We have previously demonstrated that RKIP was down-regulated in gliomas [4], and therefore, RKIP would also determine downregulation of NCL through this pathway. In contrast, NPM1 which is up-regulated in glioma [4] physically interacts with NCL and increases its activity [38]. On the other hand, HSPB1/HSP27 also up-regulates NCL, by binding to STAT3 [39], and ultimately, STAT3 may bind to NCL promoter [40], leading to cell proliferation. HSPB1/HSP27 may also activate NCL through SP1 [41], as NCL has been reported to physically interact with SP1 in non-small cell lung cancer [42], and human epidermal keratinocytes [43]. This NCL activation by HSPB1/HSP27 may receive reinforcement through GRP78, which physically interacts with ESR1 increasing its activity [44], which in turn also activates HSPB1/HSP27 promoter [45]. Therefore, HSPB1, GRP78 and EGFR may be linked through activation of two transcription factors, ESR1 and STAT3, and through a third transcription factor, SP1, this loop may be linked to RKIP, NCL and NPM1.
The present findings of HSPB1 expression enhancement with the tumor malignant progression, and NCL high expression in astrocytomas are in accordance with previously demonstrated differential expression of GRP78, NPM1 [4, 6] and EGFR [46] according to the malignant grade of astrocytomas, corroborating the relevance of this network in the progression of astrocytomas.

The combined methodology applied herein not only confirmed the relevance and the involvement of the molecular triad, NPM1, GRP78 and RKIP with glioma malignancy, but additionally has uncovered two new important targets: NOVA1, useful for the diagnostic refinement between astocytoma and oligodendroglioma, and HSPB1, as a predictive factor of poor prognosis for glioblastoma.

\section{Conclusions}

Taken together, our quantitative proteomic analysis detected the molecular triad, NPM1, GRP78 and RKIP participating together with NCL and HSP27/HSPB1 in a network related to tumor progression. Additionally, two 
new important targets were uncovered: NOVA1 useful for diagnostic refinement differentiating astrocytoma from oligodendroglioma, and HSPB1/HSP27, as a predictive factor of poor prognosis for GBM.

\section{Additional files}

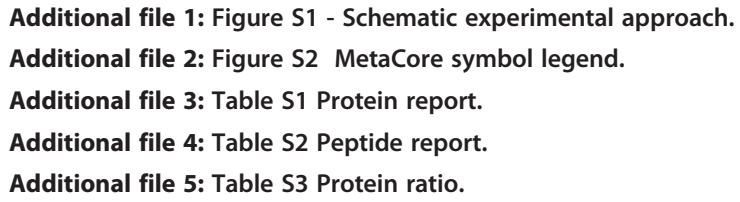

\section{Abbreviations}

ACN: Acetonitrile; AST II: AST II astrocytoma grade II; CHAPS: 3-[(3-

Cholamidopropyl)dimethylammonio]-1-propanesulfonate; DTT: Dithiothreitol; FA: Formic acid; FDR: False discovery; GBM: Glioblastoma; GBM-

LS: Glioblastoma, long survival, more than 16 months; GBM-SS: Glioblastoma, short survival, less than 12 months; GRP78: Glucose regulated protein $78 \mathrm{kDa}$; GUS: Beta-glucuronidase gene; HPRT: Hypoxanthine phosphoribosyltransferase 1 gene; HSP90B1: Heat shock protein 90 kDa; iTRAQ: Isobaric tags for relative and absolute quantitation; MS/MS: Tandem mass spectrometry; NCL: Nucleolin; NN: Non-neoplastic brain tissues; NOVA1: RNA binding protein nova 1; NPM1: Nucleophosmin; OLI II: Oligodendrogliomas grade II; OLI III: Oligodendrogliomas grade III; PCV: Procarbazin, 1-(2-cloroethyl)-3-cyclohexil-L-nitrosurea; qRTPCR: Quantitative real-time PCR; RKIP/PEBP1: Raf kinase inhibitor protein; SCX: Strong cation exchange fractionation; SDS-PAGE: Sodium dodecyl sulfate polyacrylamide gel electrophoresis; TBP: TATA-box binding protein gene; Tris: Tris(hydroxymethyl)aminomethane).

\section{Competing interests}

The authors declare that they have no competing interests.

\section{Authors' contributions}

Conceived and designed the research studies: SKM, JCR. Performed the experiments: MG, SO-S,MU, Cl. Analyzed and interpreted the data: MG, SO-S, MU, Cl, SKM, JBO, JCR. Contributed with reagents/materials/analysis tools: SKM, JBO, JCR. Drafted the manuscript: SKM, MG, JCR. Pathological revision: SKM, SO-S. All authors have read and approved the final manuscript.

\section{Acknowledgements}

We thank for technical assistance Maria Helena Dias Martins and for graphical art Sandra Navarro Bresciani (Fundação Hemocentro de Ribeirão Preto). This work was supported by the São Paulo Research Foundation (FAPESP, grant 2001/00422-5; 2004/12133-6; 2011/07568-7; 2013/02162-8, 2013/06315-3) and the National Council of Research (CNPq, Universal 487105/2012-5; 483467/ 2011-1) and productive CNPq fellowship to JCR: 304627/2012-7 and SKM: 309356/2011-3).

\section{Author details \\ 'Department Molecular and Cell Biology and Protein Chemistry Center, CTC-Center for Cell Therapy-CEPID-FAPESP-Hemocentro de Ribeirão Preto, Ribeirão Preto Medical School, University of São Paulo, São Paulo, Brazil. ${ }^{2}$ Department of Neurology, São Paulo Medical School, University of Sao Paulo, Av. Bandeirantes, 3900-14049-900 Ribeirão Preto, São Paulo, Brazil. ${ }^{3}$ Instituto de Medicina Integral Prof. Fernando Figueira-IMIP, Pernambuco, Brazil. ${ }^{4}$ Center for Studies of Cellular and Molecular Therapy (NETCEM) University of Sao Paulo, São Paulo, Brazil.}

Received: 5 December 2014 Accepted: 26 May 2015

Published online: 25 June 2015

\section{References}

1. Louis DN, Ohgaki H, Wiestler OD, Cavanee WK, editors. WHO classification of tumors of the central nervous system. Lyon: International Agency for Research on Cancer; 2007.
2. Stupp R, Hegi ME, Mason WP, van den Bent MJ, Taphoorn MJ, Janzer RC, et al. Effects of radiotherapy with concomitant and adjuvant temozolomide versus radiotherapy alone on survival in glioblastoma in a randomised phase III study: 5-year analysis of the EORTC-NCIC trial. Lancet Oncol. 2009;10(5):459-66.

3. Ohgaki H, Kleihues P. Genetic alterations and signaling pathways in the evolution of gliomas. Cancer Sci. 2009;100:2235-41.

4. Gimenez M, Souza VC, Izumi C, Barbieri MR, Chammas R, Oba-Shinjo SM, et al. Proteomic analysis of low- to high-grade astrocytomas reveals an alteration of the expression level of raf kinase inhibitor protein and nucleophosmin. Proteomics. 2010;10(15):2812-21.

5. Gimenez M, Marie SK, Oba-Shinjo SM, Uno M, da Silva R, Laure HJ, et al. Quantitative proteomic analysis and functional studies reveal that nucleophosmin is involved in cell death in glioblastoma cell line transfected with siRNA. Proteomics. 2012;12(17):2632-40.

6. Ramão A, Gimenez M, Laure HJ, Izumi C, Vida RC, Oba-Shinjo S, et al. Changes in the expression of proteins associated with aerobic glycolysis and cell migration are involved in tumorigenic ability of two glioma cell lines. Proteome Sci. 2012;10(1):53.

7. Okamoto Y, Di Patre PL, Burkhard C, et al. Population-based study on incidence, survival rates, and genetic alterations of low-grade diffuse astrocytomas and oligodendrogliomas. Acta Neuropathol (Berl). 2004;108(1):49-56.

8. Bianco AM, Miura FK, Clara C, Almeida JR, Silva CC, Teixeira MJ, et al. Low-grade astrocytoma: surgical outcomes in eloquent versus non-eloquent brain areas. Arq Neuropsiquiatr. 2013;71(1):31-4.

9. Kouwenhoven MC, Kros JM, French PJ, et al. 1p/19q loss within oligodendroglioma is predictive for response to first line temozolomide but not to salvage treatment. Eur J Cancer. 2006;42(15):2499-503.

10. Hoang-Xuan K, Capelle L, Kujas M, et al. Temozolomide as initial treatment for adults with low-grade oligodendrogliomas or oligoastrocytomas and correlation with chromosome 1p deletions. J Clin Oncol. 2004;22(15):3133-8.

11. Cairncross G, Macdonald D, Ludwin S, et al. Chemotherapy for anaplastic oligodendroglioma. National cancer institute of Canada clinical trials group. J Clin Oncol. 1994;12(10):2013-21.

12. Van den Bent MJ, Kros JM, Heimans JJ, et al. Response rate and prognostic factors of recurrent oligodendroglioma treated with procarbazine, CCNU, and vincristine chemotherapy. Dutch neuro-oncology group. Neurology. 1998;51(4):1140-5.

13. Van den Bent MJ. Guidelines for the treatment of oligodendroglioma: an evidence-based medicine approach. Forum (Genova). 2003;13(1):18-31.

14. Kesari S, Schiff D, Drappatz J, La Frankie D, Doherty L, Macklin E, et al. Phase II study protracted daily temozolomide for low- grade gliomas in adults. Clinic Cancer Res. 2009;15(1):330-7.

15. Cairncross G, Berkey B, Shaw E, et al. Phase III trial of chemotherapy plus radiotherapy compared with radiotherapy alone for pure and mixed anaplastic oligodendroglioma: intergroup radiation therapy oncology group trial 9402. J Clin Oncol. 2006;24(18):2707-14.

16. Capelle L, Oei P, Teoh H, Hamilton D, Palmer D, Low I, et al. Retrospective review of prognostic factors, including $1 \mathrm{p} 19 \mathrm{q}$ deletion, in low-grade oligodendrogliomas and a review of recent published works. J Med Imaging Radiat Oncol. 2009:53:305-9.

17. Giannini C, Scheithauer BW, Weaver AL, et al. Oligodendrogliomas: reproducibility and prognostic value of histologic diagnosis and grading. J Neuropathol Exp Neurol. 2001;60(3):248-62.

18. Sasaki H, Zlatescu MC, Betensky RA, et al. Histopathological-molecular genetic correlations in referral pathologist-diagnosed low-grade "oligodendroglioma". J Neuropathol Exp Neurol. 2002;61(1):58-63.

19. Bradford MM. A rapid and sensitive method for the quantitation of microgram quantities of protein utilizing the principle of protein-dye binding. Anal Biochem. 1976;72:248-54.

20. Keller A, Nesvizhskii Al, Kolker E, Aebersold R. Empirical statistical model to estimate the accuracy of peptide identifications made by MS/MS and database search. Anal Chem. 2002;74(20):5383-92.

21. Nesvizhskii Al, Keller A, Kolker E, Aebersold R. A statistical model for identifying proteins by tandem mass spectrometry. Anal Chem. 2003;75(17):4646-58.

22. Valente V, Teixeira SA, Neder L, Okamoto OK, Oba-Shinjo SM, Marie SK, et al. Selection of suitable housekeeping genes for expression analysis in glioblastoma using quantitative RT-PCR. BMC Mol Biol. 2009;10:17.

23. Livak KJ, Schmittgen TD. Analysis of relative gene expression data using real-time quantitative PCR and the 2(-Delta Delta C(T)) method. Methods. 2001;25 Suppl 4:402-8. 
24. Uno M, Oba-Shinjo SM, Silva Rd, Miura F, Clara CA, Almeida JR, Malheiros SM, Bianco AM, Brandt R, Ribas GC, Feres H, Dzik C, Rosemberg S, Stavale JN, Teixeira MJ, Marie SK. IDH1 mutations in a Brazilian series of Glioblastoma. Clinics (Sao Paulo). 2011;66(1):163-5

25. Uno M, Oba-Shinjo SM, Camargo AA, Moura RP, Aguiar PH, Cabrera HN, et al. Correlation of MGMT promoter methylation status with gene and protein expression levels in glioblastoma. Clinics (Sao Paulo). 2011;66(10):1747-55.

26. Mallinjoud P, Villemin JP, Mortada H, Polay Espinoza M, Desmet FO, Samaan $\mathrm{S}$, et al. Endothelial, epithelial, and fibroblast cells exhibit specific splicing programs independently of their tissue of origin. Genome Res. 2014;24(3):511-21.

27. Xu X, Joh HD, Pin S, Schiller NI, Prange C, Burger PC, et al. Expression of multiple larger-sized transcripts for several genes in oligodendrogliomas: potential markers for glioma subtype. Cancer Lett. 2001;171(1):67-77.

28. Arrigo AP, Gibert B. HspB1, HspB5 and HspB4 in human cancers: potent oncogenic role of some of their client proteins. Cancers (Basel). 2014;6(1):333-65

29. Garrido C, Ottavi P, Fromentin A, Hammann A, Arrigo AP, Chauffert B, et al. HSP27 as a mediator of confluence-dependent resistance to cell death induced by anticancer drugs. Cancer Res. 1997;57(13):2661-7.

30. Kanagasabai R, Karthikeyan K, Vedam K, Qien W, Zhu Q, Ilangovan G. Hsp27 protects adenocarcinoma cells from UV-induced apoptosis by Akt and p21-dependent pathways of survival. Mol Cancer Res. 2010;8(10):1399-412.

31. Marquez DC, Lee J, Lin T, Pietras RJ. Epidermal growth factor receptor and tyrosine phosphorylation of estrogen receptor. Endocrine. 2001;16(2):73-81.

32. Park S, Yeung ML, Beach S, Shields JM, Yeung KC. RKIP downregulates B-Raf kinase activity in melanoma cancer cells. Oncogene. 2005;24(21):3535-40.

33. Zoncu R, Efeyan A, Sabatini DM. mTOR: from growth signal integration to cancer, diabetes and ageing. Nature Rev Mol Cell Biol. 2011;12(1):21-35.

34. Chen B, Tardell C, Higgins B, Packman K, Boylan JF, Niu H. BRAFV600E negatively regulates the AKT pathway in melanoma cell lines. PloS One. 2012;7(8), e42598.

35. Okumura E, Fukuhara T, Yoshida H, Hanada Si S, Kozutsumi R, Mori M, et al. Akt inhibits Myt1 in the signalling pathway that leads to meiotic G2/M-phase transition. NatCell Biol. 2002;4(2):111-6.

36. Hutchins JR, Toyoda Y, Hegemann B, Poser I, Heriche JK, Sykora MM Augsburg M, Hudecz O, Buschhorn BA, Bulkescher J, Conrad C, Comartin D, Schleiffer A, Sarov M, Pozniakovsky A, Slabicki MM, Schloissnig S, Steinmacher I, Leuschner M, Ssykor A, Lawo S, Pelletier L, Stark H, Nasmyth K, Ellenberg J, Durbin R, Buchholz F, Mechtler K, Hyman AA, Peters JM. Systematic analysis of human protein complexes identifies chromosome segregation proteins. Science (New York, N.Y.). 2010;328(5978):593-99.

37. Wang SA, Li HY, Hsu TI, Chen SH, Wu CJ, Chang WC, et al. Heat shock protein 90 stabilizes nucleolin to increase mRNA stability in mitosis. J Biol Chem. 2011;286(51):43816-29.

38. Maggi Jr LB, Kuchenruether M, Dadey DY, Schwope RM, Grisendi S, Townsend RR, et al. Nucleophosmin serves as a rate-limitingnuclear export chaperone for the mammalian ribosome. Mol Cellular Biol. 2008;28(23):7050-65.

39. Rocchi P, Beraldi E, Ettinger S, Fazli L, Vessella RL, Nelson C, et al. Increased Hsp27 after androgen ablation facilitates androgen-independent progression in prostate cancer via signal transducers and activators of transcription 3-mediated suppression of apoptosis. Cancer Res. 2005;65(23):11083-93.

40. Beck HC, Petersen J, Nielsen SJ, Morsczeck C, Jensen PB, Sehested M, et al. Proteomic profiling of human colon cancer cells treated with the histone deacetylase inhibitor belinostat. Electrophoresis. 2010;31(16):2714-21.

41. Friedman MJ, Li S, Li XJ. Activation of gene transcription by heat shock protein 27 may contribute to its neuronal protection. J Biol Chem. 2009;284(41):27944-51.

42. Tsou JH, Chang KY, Wang WC, Tseng JT, Su WC, Hung LY, et al. Nucleolin regulates C-Jun/Sp1-dependent transcriptional activation of CPLA2alpha in phorbol ester-treated non-small cell lung cancer A549 cells. Nucleic Acids Res. 2008;36(1):217-27.

43. Sakaguchi M, Miyazaki M, Takaishi M, Sakaguchi Y, Makino E, Kataoka N, et al. S100C/A11 is a key mediator of $\mathrm{Ca}(2+)$-induced growth inhibition of human epidermal keratinocytes. J Cell Biol. 2003;163(4):825-35.

44. Ray S, Hou X, Zhou HE, Wang H, Das SK. Bip is a molecular link between the phase I and phase II estrogenic responses in uterus. Mol Endocrinol (Baltimore, Md.). 2006;20(8):1825-37.
45. Carroll JS, Meyer CA, Song J, Li W, Geistlinger TR, Eeckhoute J, et al. Genome-wide analysis of estrogen receptor binding sites. Nature Gen. 2006;38(11):1289-97.

46. Carvalho PO, Uno M, Oba-Shinjo SM, Rosemberg S, Wakamatsu A, da Silva CC, et al. Activation of EGFR signaling from pilocytic astrocytomas to glioblastomas. Int J Biol Markers. 2014:29(2):e120-8.

\section{Submit your next manuscript to BioMed Central and take full advantage of:}

- Convenient online submission

- Thorough peer review

- No space constraints or color figure charges

- Immediate publication on acceptance

- Inclusion in PubMed, CAS, Scopus and Google Scholar

- Research which is freely available for redistribution 